FACTA UNIVERSITATIS

Series: Mechanical Engineering Vol. 15, No 3, 2017, pp. 535 - 543

https://doi.org/10.22190/FUME170504021Z

Original scientific paper

\title{
COMPUTERIZED RADIAL ARTERY PULSE SIGNAL CLASSIFICATION FOR LUNG CANCER DETECTION
}

\author{
UDC 004.8:616
}

\begin{abstract}
Zhichao Zhang ${ }^{1,2}$, Anton Umek ${ }^{2}$, Anton Kos ${ }^{2}$
${ }^{1}$ Shandong Provincial Key Laboratory of Network Based Intelligent Computing, University of Jinan, Jinan, China

${ }^{2}$ Faculty of Electrical Engineering, University of Ljubljana, Slovenia

Abstract. Pulse diagnosis, the main diagnosis method in traditional Chinese medicine, is a non-invasive and convenient way to check the health status. Doctors usually use three fingers to feel three positions; Cun, Guan, and Chi of the wrist pulse, to diagnose the body's healthy status. However, it takes many years to master the pulse diagnosis. This paper aims at finding the best position for acquiring wrist-pulse-signal for lung cancer diagnosis. In our paper, the wrist-pulse-signals of Cun, Guan, and Chi are acquired by three optic fiber pressure sensors of the same type. Twelve features are extracted from the signals of these three positions, respectively. Eight classifiers are applied to detect the effectiveness of the signal acquired from each position by classifying the pulse signals of healthy individuals and lung cancer patients. The results achieved by the proposed features show that the signal acquired at Cun is more effective for lung cancer diagnosis than the signals acquired at Guan and Chi.
\end{abstract}

Key Words: Radial Artery Pulse Feature Extraction, Pulse Signal Classification, Lung Cancer Detection

\section{INTRODUCTION}

Lung cancer is one of the major public health problems worldwide [1]. Pulse diagnosis is a safe and auxiliary way of detecting lung cancer in clinical medicine [2]. Since pulse diagnosis heavily depends on physicians' experience, it takes a very long time and a lot of energy to master the technique of pulse diagnosis [3]. With the development of sensor technology, signal processing, data analysis techniques, and artificial intelligence, the pulse based diagnosis can be implemented by processing the radial artery pulse signal $[4$, 5]. To popularize the pulse diagnosis in clinical medicine, researchers have been trying to

Received May 04, 2017 / Accepted October 31, 2017

Corresponding author: Anton Umek

Faculty of Electrical Engineering, University of Ljubljana, Tržaška c. 25, 1000 Ljubljana, Slovenia

E-mail: anton.umek@fe.uni-lj.si 
acquire and process the radial artery pulse signal using sensor technology and machine learning [6-7].

Yong Jun et al. [8] designed a sensor from the resonator to acquire the radial artery pulse signal based on the reflection coefficient; the sensor can be embedded in a wearable communication device. According to their experiment results, the acquired signal reflects the useful artery information and meets the requirements of the signal analysis for clinical purposes. Zuo et al. [9] analyzed three types of radial artery sensors: pressure, photoelectric, and ultrasonic sensors according to the physical meanings, correlations of the acquired signal from these three sensors, and sensitivity factors. Their conclusion shows: it is better to measure the transmural pressure to diagnose some special disease which can change the elastic property and thickness of the vessel wall. According to the pulse diagnosis theory [10], the lung cancer patients' vessel wall is different from that of healthy individuals; thus the signal of pulse pressure acquired from the optical sensor in our experiment has useful information for detecting lung cancer.

Machine learning is a popular and effective way of analyzing the radial artery pulse signal. Khaire et al. [11] extracted the spectral features from the radial artery pulse signal and used the support vector machine to classify the individuals' pre-meal and post-meal signals. In Rangaprakash's experiment [12], the pulse signal was taken from the volunteers before and after exercise. Seven spatial features extracted from the pulse wave are used to classify the signals into these two groups using Recursive Cluster Elimination based Support Vector Machine. Their work proved that the features obtained from the radial artery pulse signal can be used to detect the physiological state of the individuals.

Zhang and Sun [13] utilize a convolutional neural network to classify twelve pulse waveforms; long pulse, feeble pulse, stringy pulse, thready pulse, deep pulse, rapid pulse, hesitant pulse, soft pulse, short pulse, slippery pulse, flood pulse, and faint pulse. Half of the dataset with 200 samples was selected to train the classifier without feature extraction and the classification accuracy was found to be $93.49 \%$. However, this is the basic traditional wrist pulse waveform classification which could not be used to disease diagnosis.

Chow et al. [14] defined the Doppler parameters to be the disease sensitive features and applied the Support Vector Machine to distinguish between Acute Appendicitis patients and healthy individuals. Gong et al. [15] designed a wrist pulse sensing and analyzing system for recognition of cirrhosis patients with an accuracy of $87.09 \%$. Unlike the work they did, our research aims to identify lung cancer patients from healthy individuals.

Currently, in the radial-artery-signal processing research, only few works are directly related to the detection of some specific disease. To the best of our knowledge, no related work uses a radial artery pulse signal to identify lung cancer patients. In this work, we extract the features from the radial artery pulse signal for identification of lung cancer patients' and compare the effectiveness of the wrist-pulse-signals acquired from the Cun, Guan and Chi positions as shown in Fig. 1c.

The rest of our paper is organized as follows: Section II briefly explains the data acquisition and pre-processing. The proposed features for lung cancer detection are listed in Section III. Section IV presents and discusses the experiment result, and Section V concludes the paper. 


\section{DATA ACQUISITION AND PRE-PROCESSING}

The dataset is acquired from the Shandong Academy of Chinese Medicine. Fig. 1a shows the pulse signal acquisition device. The signal sampling rate is $800 \mathrm{~Hz}$. The raw continuous signal was collected from the Cun, Guan, and Chi wrist locations of 16 healthy individuals and 15 lung cancer patients. Different levels of pressure are imposed on the radial artery by moving the robotic arm during the acquisition of the radial pulse signal. An example of a raw continuous signal with five pressure levels is shown in Fig. 1b. The locations of "Cun" and "Guan" and "Chi" of the wrist are shown in Fig.1c. Figure 2a shows the enlarged local signal at one pressure level.

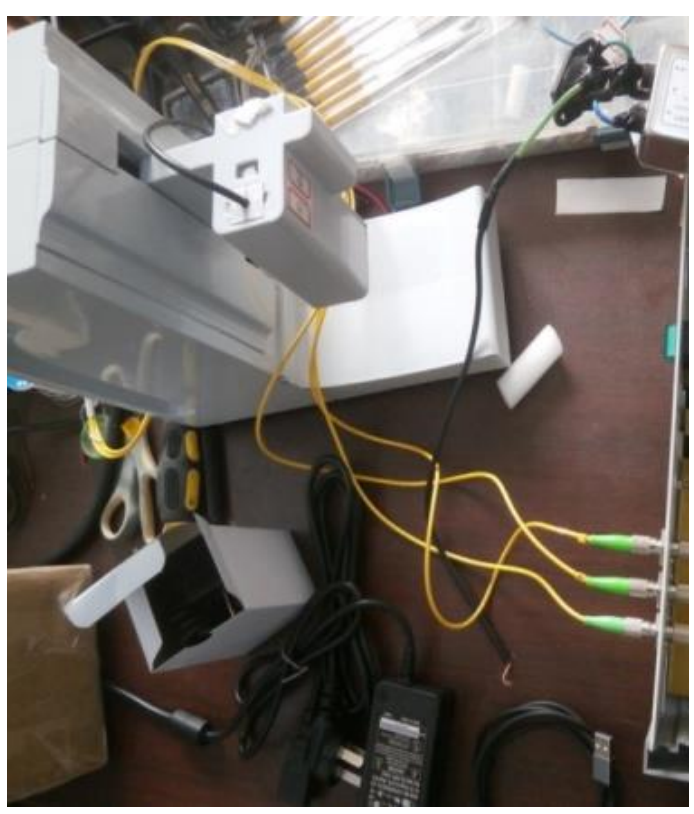

a)

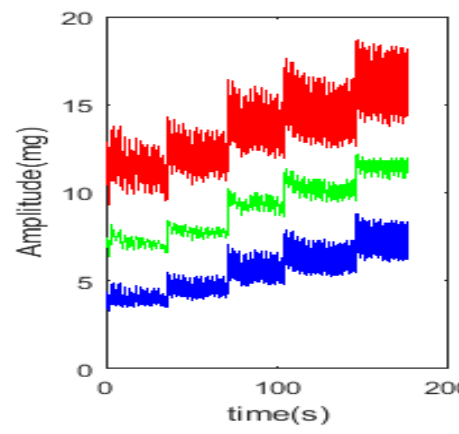

b)

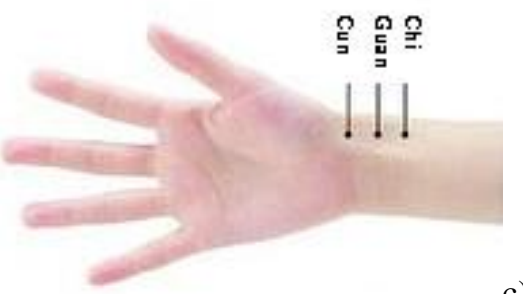

c)

Fig. 1 a) The pulse acquisition device; b) The acquired raw signal;

c) The locations of Cun, Guan and Chi

In our previous work, we utilized the Gaussian filter to de-noise the signal and designed an algorithm to remove the baseline wander and segment the continuous signal into single periods of the signal. Fig. $2 b$ shows the de-noised signal with the fitted baseline. The corresponding segmentation result is shown in Fig. 2c. From Fig. 2, it is evident that the baseline is removed and the single periods of the signal are segmented successfully using the developed algorithm. In addition, the average value of baseline is recorded to be used as one of the features in our experiment.

After segmentation, we extracted features from the single periods and used classifiers to recognize the valid single periods of the signal. The examples of invalid and valid single wrist-pulse-signal periods are shown in Fig. 3a to Fig. 3d, respectively. Finally, 8508 valid single periods of the radial-artery-signal are available for classification. 


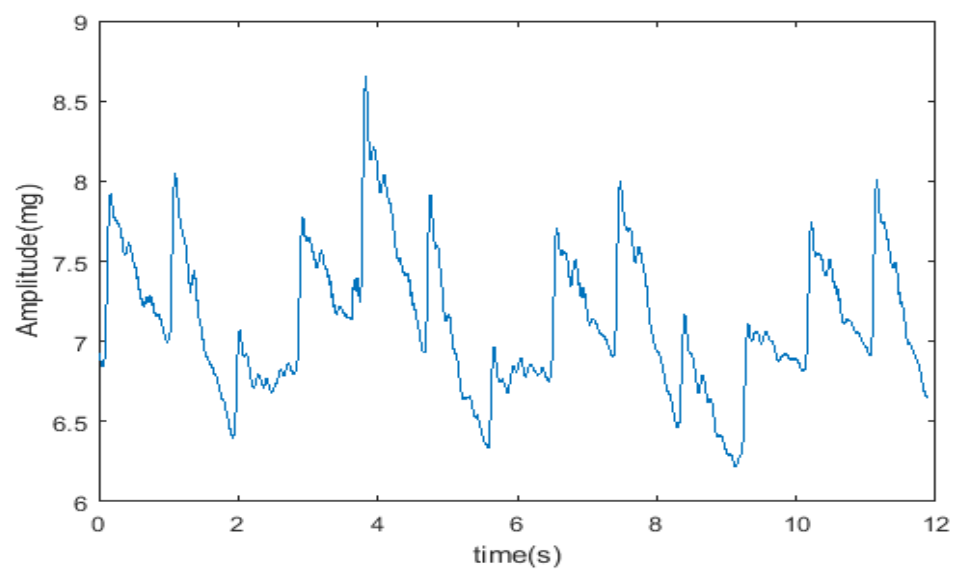

a)

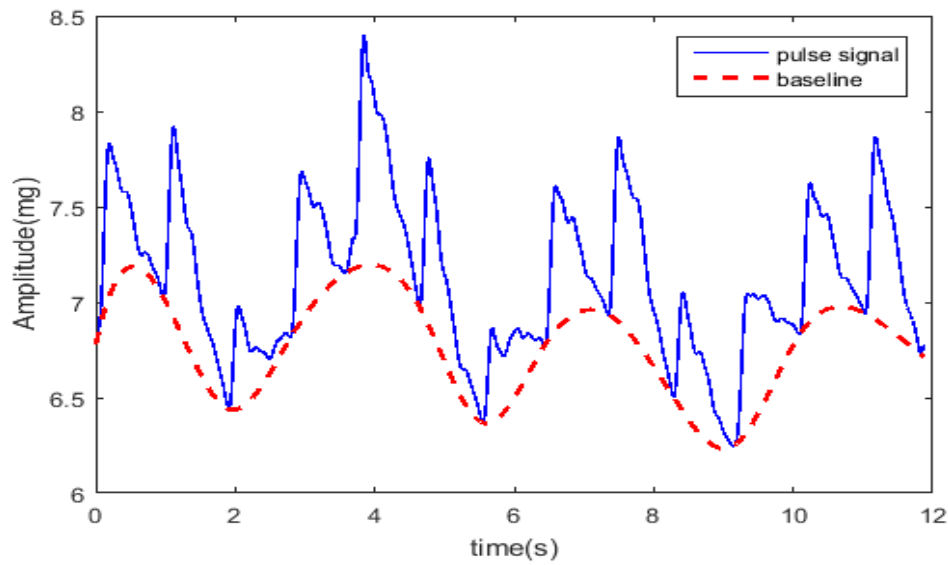

b)

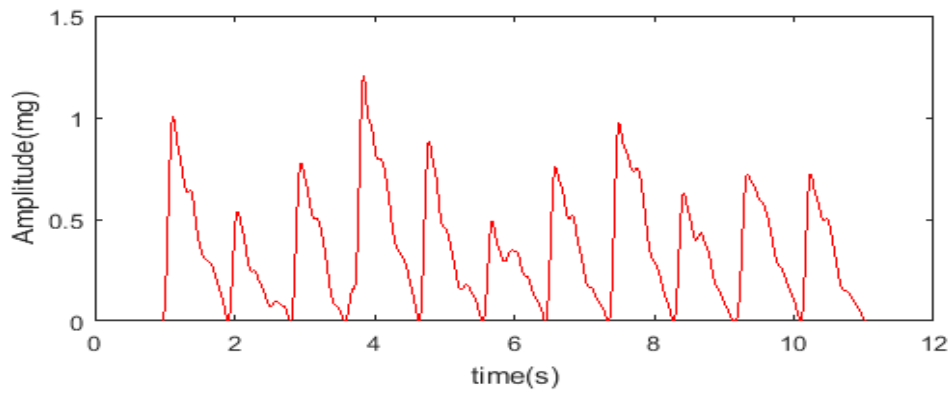

c)

Fig. 2 a) An example of the enlarged raw signal; b) An example of the baseline fitting result; c) An example of the segmentation result 


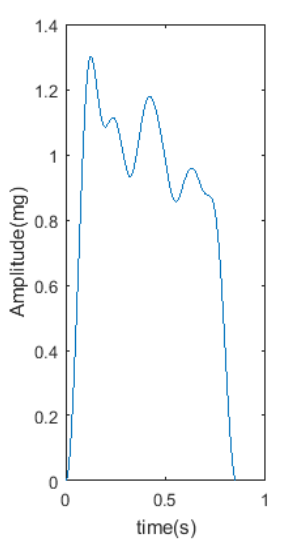

a)

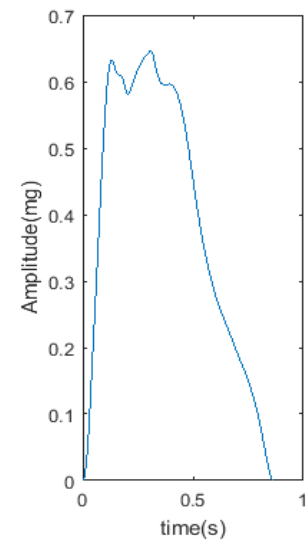

b)

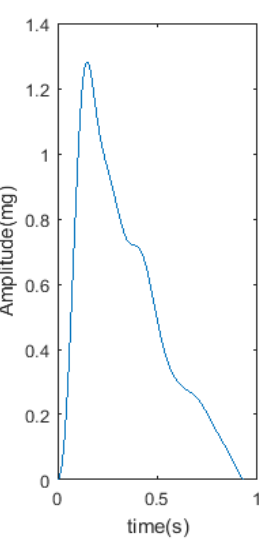

c)

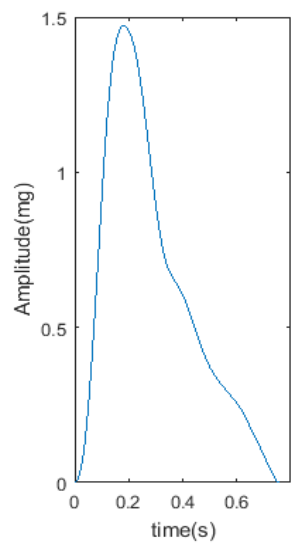

d)

Fig. 3 a) and b) Examples of invalid periods; c) and d) Examples of valid periods

\section{FEATURE EXTRACTION}

In our paper, we analyze single periods of the radial-artery-signal in the time domain. The features are extracted from the shapes of single periods. In Fig. 4, it can be seen that the single period has a cardiac rapid ejection part and a heart slow-firing blood part (SFBP). The rapid ejection period lasts from the start of the period to the maximum. And SFBP lasts from the maximum to the end of the period. For more convenient features extraction, the SFBP is divided into 5 parts (a1, a2, a3, b1, b2) in the time domain, as shown in Fig. 4. The parts of a1, a2, a3 account 1/8 respectively of SFBP, whereas b1 and b2 account $1 / 4$ and 3/8 of SFBP, respectively.

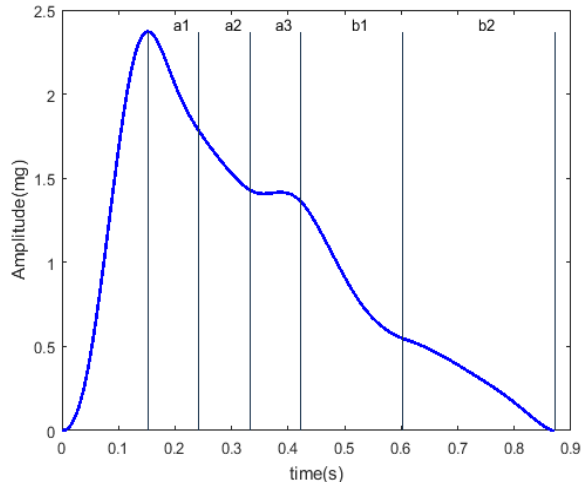

a)

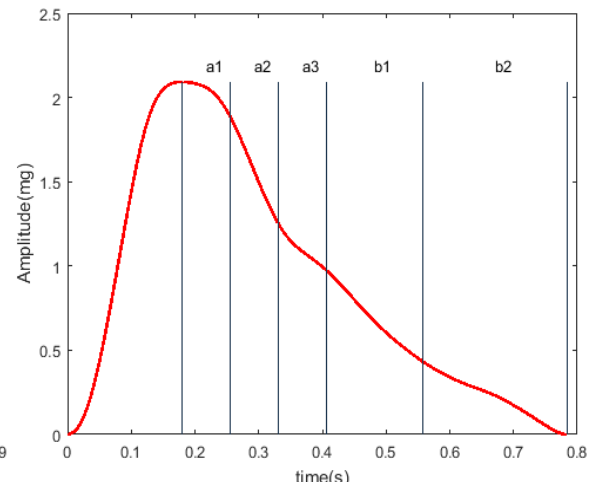

b)

Fig. 4 The single period radial-artery-signals: a) Signal of the healthy individual, b) Signal of the lung cancer patient 
The features are extracted from different parts of the period. The twelve features shown in Table 1 are used to train the classifiers and identify the lung cancer patients' signals.

Table 1 Definitions of features

\begin{tabular}{ll}
\hline Name & Definition \\
\hline pressure & The depth of probe descent \\
ave_single & Average baseline value of the single period of wrist pulse signal \\
len_period & Length of the single period of wrist-pulse-signal \\
pmax_index & Index of the sample of the maximum \\
num_09 & Number of points that fit the condition (maximum-minimum >= 0.9) \\
ave_a1 & Average value of a signal in b1 clip \\
ave_a2 & Average value of a signal in b2 clip \\
ave_a3 & Average value of a signal in b3 clip \\
ave_b1 & Average value of a signal in c1 clip \\
ave_b2 & Average value of a signal in c2 clip \\
min_psub & The distance between the peaks of heart slow-firing blood period \\
location & Signal acquisition location (Cun, Guan, Chi) \\
\hline
\end{tabular}

The feature pressure represents different pressure levels imposed on the volunteers' radial artery. When changing the imposed pressure, the baseline of the signal changes as well. Thus, we calculate the baseline's average value (ave_single) to be a feature as well. The length of the period (len_period) is given in seconds, and the average values of a1, a2, $\mathrm{a} 3, \mathrm{~b} 1$, and b2 are extracted as features. A lung cancer patient's cardiac rapid ejection period is longer than that of a healthy individual as shown in Fig.4. Accordingly, we extract the index of the maximum amplitude (pmax_index). In addition, the lung cancer patients' heart slow-firing blood period is different in shape from that of healthy individuals. Feature min_psub is the distance between the peaks in heart slow-firing blood period. Since the radial-artery-signal is acquired from three probes on Cun, Guan, and Chi, the acquisition location is also taken as a feature.

\section{ClassiFICATION}

In this experiment, we have three different training sets. Each training set consists of signals acquired from two healthy individuals and two lung cancer patients from one of the three positions, i.e. Cun, Guan, and Chi. The rest of the individuals' data forms the three corresponding testing sets. At the extraction of the aforementioned features, the following classifiers are used to classify the periods of healthy individuals and lung cancer patients: Linear SVM (support vector machine), Coarse Gaussian SVM, Fine KNN (K-nearest neighbors), Cosine KNN, Subspace discriminant, Subspace KNN, Simple Tree and Quadratic discriminant. Each individual's classification results are counted respectively to calculate the lung cancer case rate. In this experiment, the one whose lung cancer periods are accounted for $50 \%$ or more is diagnosed to be a lung cancer patient.

Table 2 shows the diagnosis accuracy of lung cancer patients and healthy individuals using the abovementioned classifiers. Each individual's diagnosis results coupled with the best classification accuracy are shown in Table 3. 
Table 2 Diagnosis accuracy of different classifiers

\begin{tabular}{lccc}
\hline Classifier & \multicolumn{3}{c}{ Diagnosis Accuracy } \\
\cline { 2 - 4 } & Cun & Guan & Chi \\
\hline Linear SVM & $88.46 \%$ & $80.00 \%$ & $88.00 \%$ \\
Coarse Gaussian SVM & $96.15 \%$ & $88.00 \%$ & $88.00 \%$ \\
Fine KNN & $96.15 \%$ & $68.00 \%$ & $88.00 \%$ \\
Cosine KNN & $96.15 \%$ & $72.00 \%$ & $88.00 \%$ \\
Subspace discriminant & $88.46 \%$ & $84.00 \%$ & $76.00 \%$ \\
Subspace KNN & $65.38 \%$ & $64.00 \%$ & $88.00 \%$ \\
Simple tree & $73.08 \%$ & $64.00 \%$ & $84.00 \%$ \\
Quadratic discriminant & $73.08 \%$ & $76.00 \%$ & $84.00 \%$ \\
\hline
\end{tabular}

Table 3 Diagnosis accuracy for individual subjects

\begin{tabular}{ccccl}
\hline Individual & Cun & Guan & Chi & Label \\
\hline 1 & $32.26 \%$ & $82.61 \%$ & $50.00 \%$ & Healthy \\
2 & $47.14 \%$ & $96.50 \%$ & $37.63 \%$ & Healthy \\
3 & $81.98 \%$ & $66.67 \%$ & $34.78 \%$ & Healthy \\
4 & $0.00 \%$ & $7.06 \%$ & $50.00 \%$ & Healthy \\
5 & $0.00 \%$ & $4.52 \%$ & $10.15 \%$ & Healthy \\
6 & $0.00 \%$ & $42.78 \%$ & $6.83 \%$ & Healthy \\
7 & $0.00 \%$ & $17.56 \%$ & $7.97 \%$ & Healthy \\
8 & $0.00 \%$ & $17.56 \%$ & $7.97 \%$ & Healthy \\
9 & $0.00 \%$ & $45.51 \%$ & $4.23 \%$ & Healthy \\
10 & $0.00 \%$ & $38.64 \%$ & $20.00 \%$ & Healthy \\
11 & $0.00 \%$ & $34.78 \%$ & $0.00 \%$ & Healthy \\
12 & $0.00 \%$ & $0.00 \%$ & $0.00 \%$ & Healthy \\
13 & $0.63 \%$ & $0.00 \%$ & $3.23 \%$ & Healthy \\
14 & $0.00 \%$ & $0.00 \%$ & $0.63 \%$ & Healthy \\
15 & $100.00 \%$ & $100.00 \%$ & insufficient & Lung cancer \\
16 & $100.00 \%$ & insufficient & $100.00 \%$ & Lung cancer \\
17 & $100.00 \%$ & $100.00 \%$ & $100.00 \%$ & Lung cancer \\
\hline 18 & $80.00 \%$ & $100.00 \%$ & $100.00 \%$ & Lung cancer \\
19 & insufficient & $100.00 \%$ & $0.00 \%$ & Lung cancer \\
\hline 20 & $100.00 \%$ & insufficient & insufficient & Lung cancer \\
21 & $100.00 \%$ & $100.00 \%$ & $100.00 \%$ & Lung cancer \\
\hline 22 & $100.00 \%$ & $100.00 \%$ & $100.00 \%$ & Lung cancer \\
23 & $100.00 \%$ & $100.00 \%$ & $83.33 \%$ & Lung cancer \\
24 & $100.00 \%$ & $99.50 \%$ & $53.96 \%$ & Lung cancer \\
25 & $100.00 \%$ & $100.00 \%$ & $100.00 \%$ & Lung cancer \\
26 & $100.00 \%$ & $100.00 \%$ & $87.50 \%$ & Lung cancer \\
27 & $90.48 \%$ & $92.03 \%$ & $71.62 \%$ & Lung cancer \\
\hline
\end{tabular}

Note: The result of 'insufficient' means the samples of the corresponding individual are not enough for classification 


\section{DISCUSSION}

As shown in Table 2, the best result for the signal acquired from Guan is $88.00 \%$, and the corresponding classifier is Coarse Gaussian SVM. While the best diagnosis accuracy of $88.00 \%$ for the pulse signal acquired from Chi is achieved by the two SVM models and the three KNN models mentioned above. Compared with the signal acquired from Chi and Guan, the signal acquired from Cun shows the best result when using 5 classifiers (linear SVM, coarse Gaussian SVM, fine KNN, cosine KNN, subspace discriminant), especially when using coarse Gaussian SVM, fine KNN, and cosine $\mathrm{KNN}$, the diagnosis accuracy is $96.15 \%$.

In addition, we notice that Coarse Gaussian SVM performs best for the signal acquired from all these three positions. Thus, to show the performance of the coarse Gaussian SVM classifier in detail, we show each individual's diagnosis result in Table 3 for Coarse Gaussian SVM classifier. Table 3 shows that two healthy individuals (individual 1 and 4) and a lung cancer patient (individual 19) are diagnosed incorrectly with the signal acquired from Chi, whereas three healthy individuals (individual 1 , individual 2, individual 3 ) are misdiagnosed to be lung cancer patients with the signals of Guan. While, when using the signal acquired from Guan and Cun, all the lung cancer patients can be recognized correctly. There is only one healthy individual (individual 3) misdiagnosed with the signal acquired from Cun.

In summary, our proposed twelve features are effective to distinguish between the radial-artery-signal of healthy individuals and lung cancer patients, and compared with the signal acquired from Chi and Guan, the signal acquired from the position of Cun gives the most accurate classification results, especially with the coarse Gaussian SVM classifier.

In the current work, the radial-artery-signal related features are taken only from the time domain. In our future work, we plan to analyze the signal in the frequency domain by extracting its frequency domain features. Meanwhile, we will try to get more lung cancer patients' and healthy individuals' radial-artery-signals to test the effectiveness of the pulse signal acquired from Cun. Moreover, we plan to design a more effective signal preprocessing procedure and build a more effective model to get more accurate classification results.

\section{CONCLUSION}

This paper is a study of the possibilities of lung cancer patient recognition using machine learning algorithms. The wrist pulse signal is acquired from healthy individuals and from lung cancer patients. The acquisition is performed simultaneously at the radial artery locations of Cun, Guan, and Chi. In this work, we extract twelve features from the single period of signal and build eight classifiers to recognize the signal of lung cancer patients. The results show that the pulse signal acquired from Cun performs the best compared with that from Guan and Chi. The extracted features are verified to be $96.15 \%$ accurate for the lung cancer diagnosis with the signal acquired from Cun using the classifier of coarse Gaussian SVM.

In our future work, we will improve the raw signal pre-processing algorithms, obtain more raw signals, and try to find even more effective features to increase the diagnosis accuracy of lung cancer detection with the emphasis on the pulse signal acquired from the position of Cun. 
Acknowledgments: We appreciate the support given by the Shandong Academy of Chinese Medicine. This work was supported in part by the National Natural Science Foundation of China under Grant 61572231, in part by the Shandong Provincial Key Research \& Department Project under Grant 2017GGX10141, and in part by the Slovenian Research Agency within the research program Algorithms and Optimization Methods in Telecommunications. The corresponding author is Anton Umek.

\section{REFERENCES}

1. Siegel, R.L., Miller, K.D., Jemal, A., 2016, Cancer statistics, 2016, CA: a cancer journal for clinicians, 66(1), pp. 7-30.

2. Luo, C.H., Su, C.J., Huang, T.Y., Chung, C.Y., 2016, Non-invasive holistic health measurements using pulse diagnosis: I. Validation by three-dimensional pulse mapping, European Journal of Integrative Medicine, 8(6), pp. 921-925.

3. Hsu, W.C., 2016, Method and system for detecting signals of pulse diagnosis, and detecting device of pulse diagnosis, U.S. Patent Application 14/991, pp. 407.

4. Jun, M.H., Kim, Y.M., Bae, J.H., Chang, J.J., Cho, J.H., Young, J.J., 2016, Development of a tonometric sensor with a decoupled circular array for precisely measuring radial artery pulse, Sensors, 16(6), doi: 10.3390/s16060768

5. Moura, N.G.R., Arthur S.F., 2016, Pulse waveform analysis of Chinese pulse images and its association with disability in hypertension, Journal of acupuncture and meridian studies, 9(2), pp. 93-98.

6. Sweeney, J.S., 1977, Blood pulse sensor and readout, U.S. Patent 4, 063, 551.

7. Garg, N., Ramandeep, K., Harry, G., Hardeep, S.R., Amod, K., 2017, Wrist pulse signal features extraction: virtual instrumentation, Proc. International Conference on Intelligent Communication, Control and Devices, Singapore, pp. 135-142.

8. Jun, Y., Yun, G.H., Kim, S.W., Yook, J.G., 2014, Wrist pulse detection system based on changes in the near-field reflection coefficient of a resonator, IEEE Microwave and Wireless Components Letters, 24(10), pp. 719-721.

9. Zuo, W.M., Wang, P., Zhang, D., 2016, Comparison of three different types of wrist pulse signals by their physical meanings and diagnosis performance, IEEE journal of biomedical and health informatics, 20(1), pp. 119-127.

10. Dong, H.X., Lu, P., Wang, X., 1999, Hemorheological changes and its clinical significance in patients with operable lung cancer, Journal of Chinese Microcirculation, 1999(4), pp.5.

11. Khaire, N.N., Joshi, Y.V., 2015, Diagnosis of disease using wrist pulse signal for classification of pre-meal and post-meal samples, Proc. Industrial Instrumentation and Control (ICIC), 2015 International Conference, IEEE, Pune, pp. 866-869.

12. Rangaprakash, D., Dutt, D.N., 2014, Analysis of wrist pulse signals using spatial features in time domain, Proc. Communications and Signal Processing (ICCSP), 2014 International Conference, IEEE, Melmaruvathur, pp. 345-348.

13. Zhang, S.R., Sun, Q.F., 2016, Human pulse recognition based on convolutional neural networks, Proc. Computer, Consumer and Control (IS3C), 2016 International Symposium, Xian, IEEE, pp. 366-369.

14. Chow, W.H., Wu, C.K., Tsang, K.F., Li, B.Y.S., Kwok, T.C., 2014, Wrist pulse signal classification for inflammation of appendix, pancreas, and duodenum, Proc. Industrial Electronics Society, IECON 2014-40th Annual Conference, IEEE, Dallas, pp. 2479-2483.

15. Gong, S., Xu, B., Sun, G., Chen, M., Wang, N., Dong, C., Wang, P., Cui, J., 2012, Accurate cirrhosis identification with wrist-pulse data for mobile healthcare, Proc. Second ACM Workshop on Mobile Systems, Applications, and Services for HealthCare, ACM, Toronto, pp. 6. 